\title{
Septischer Schock und systemisches Entzündungsreaktions-Syndrom
}

\section{Eine und nur eine Antwort ist jeweils richtig!}

Eine „schwere Sepsis“ unterscheidet sich von einer „Sepsis“ Iaut internationaler Definition durch..

Der Schweregrad eines septischen Multiorganversagens kann am genauesten quantifiziert werden durch...

Erster entscheidender Schritt bei der Behandlung des septischen Kreislaufversagens ist die sofortige Verabreichung von ...

Eine hämodynamisch gesteuerte Herz-KreislaufTherapie bei Patienten mit schwerer Sepsis und septischem Schock sollte begonnen werden...

Eine Thromboseprophylaxe...
A Fieber über $40^{\circ} \mathrm{C}$.

B den Nachweis mehrerer verschiedener Keime in der Blutkultur oder anderen Körperflüssigkeiten.

C das Vorliegen von Zeichen der Organdysfunktion, Minderperfusion oder Hypotonie.

D einen besonders langen und komplizierten Verlauf.

E die Notwendigkeit eines Intensivstationsaufenthaltes.

A den CRP-Spiegel.

B die Körpertemperatur.

C die Leukozytenzahl im peripheren Blut.

D Scoresysteme.

E den Interleukin-6-Spiegel.

A Volumen.

B Adrenalin.

C Antibiotika.

D Hydrocortison.

E aktiviertem Protein C.

A erst nach Vorliegen der mikrobiologischen Befunde.

$B$ bereits auf der Notaufnahmestation.

C auf der Intensivstation.

D erst nach Vorliegen des Laktatspiegels.

E erst nachdem das Echokardiogramm vorliegt.

A ist bei Sepsispatienten kontraindiziert.

$B$ ist bei Sepsispatienten nicht erforderlich.

C verbessert die Prognose des Sepsispatienten.

D ist bei Sepsispatienten noch nicht ausreichend untersucht.

E sollte nur initial im Verlauf der Sepsis durchgeführt werden. 
Kortikosteroide...

Der Hämoglobinspiegel sollte bei Patienten mit schwerer Sepsis..
A sind bei Sepsispatienten kontraindiziert.

B sollten bei Sepsispatienten hochdosiert verabreicht werden, um die Entzündung zu hemmen.

C dürfen bei Sepsispatienten nur gegeben werden, wenn in der Blutkultur keine Keime mehr nachweisbar sind.

D können bei Patienten mit refraktärem septischen Schock in moderater Dosierung zur Substitution der relativen Nebennierenrinden-Insuffizienz gegeben werden.

E dürfen bei Sepsispatienten nur gegeben werden, wenn es zu einer anaphylaktoiden Reaktion gekommen ist.

A nicht unter 7-8 g/dl sinken.

B nicht unter $11 \mathrm{~g} / \mathrm{dl}$ sinken.

C nicht unter $6 \mathrm{~g} / \mathrm{dl}$ sinken.

D nicht unter $5 \mathrm{~g} / \mathrm{dl}$ sinken.

E grundsätzlich durch die Gabe von Erythropoietin erhöht werden.

Welche Aussage ist richtig?

B Bei weniger als der Hälfte der Sepsispatienten finden sich positive Blutkulturen.

C Negative Blutkulturen schließen eine disseminierte Gewebsbesiedlung mit Keimen aus.

D Eine Sepsis wird ganz überwiegend durch Gram-negative Keime hervorgerufen.

E Eine nosokomial erworbene und eine ambulant erworbene Sepsis unterscheiden sich nicht hinsichtlich der Prognose.

Die Herdsanierung bei einem septischen Patienten...

A ist nach Entwickeln des septischen Krankheitsbildes nicht mehr sinnvoll.

$B$ ist die entscheidende kausale Therapie der Sepsis.

C erfolgt am effizientesten durch eine gezielte Antibiotikatherapie.

D ist nur bei positiven Blutkulturen erforderlich.

E hat keinen Einfluss auf den Krankheitsverlauf.

Die autonome Dysfunktion in der Sepsis kann diagnostiziert werden....

Bei einer Sepsis...
A durch Messung von Nervenleitgeschwindigkeiten.

B durch Messung des Noradrenalinblutspiegels.

C durch Bestimmung der Herzfrequenzvariabilität im EKG.

D durch einen hohen Katecholaminbedarf.

E durch erhöhten Speichelfluss des Patienten.

A kann die Körpertemperatur erniedrigt sein.

B ist die Körpertemperatur initial immer erhöht.

C findet sich initial immer eine Leukozytose.

D ist der Patient oft bradykard.

E kommt es häufig initial zu einer respiratorischen Azidose.

\section{Teilnahmebedingungen}

Für diese Fortbildungseinheit erhalten Sie drei Fortbildungspunkte im Rahmen des freiwilligen Fortbildungszertifikates. Hierfür

- müssen 10 der 11 Quiz-Fragen richtig beantwortet sein (bestanden hat auch, wer nicht weniger Fragen richtig beantwortet hat als der Durchschnitt aller Teilnehmer).

- müssen Seite 1 und Seite 2 des Evaluationsbogens vollständig ausgefüllt sein. Unvollständig ausgefüllte Bögen können nicht berücksichtigt werden!

- muss eine CME-Wertmarke im Feld D (Evaluationsbogen) aufgeklebt oder Ihre DMW-Abonnement-Nummer eingetragen sein.

\section{Datenschutz}

Ihre Daten werden ausschließlich für die Bearbeitung dieser Fortbildungseinheit verwendet. Es erfolgt keine Speicherung der Ergebnisse über die für die Bearbeitung der Fortbildungseinheit notwendige Zeit hinaus. Die Daten werden nach Versand der Testate anonymisiert. Namens- und Adressangaben dienen nur dem Versand der Testate. Die Angaben zur Person dienen nur statistischen Zwecken und werden von den Adressangaben anonymisiert verarbeitet.

\section{Einsendeschluss ist der 7.1.2004}

(Datum des Poststempels). Die Zertifikate werden ab dem 14.1.2004 auf dem Postweg versandt. Von telefonischen Anfragen bitten wir abzusehen. Die richtige Auflösung des Quiz wird in der DMW Nr. 3/2004 (erscheint am 16.1.2004) veröffentlicht. 